Port Acadie

Revue interdisciplinaire en études acadiennes

An Interdisciplinary Review in Acadian Studies

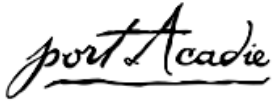

\title{
La paroisse et l'église Sacré-Coeur de Saulnierville
}

\section{Jean-Louis Robichaud}

Numéro 10-11-12, automne 2006, printemps-automne 2007

Le patrimoine religieux de la Nouvelle-Écosse : signes et paradoxes en Acadie

URI : https://id.erudit.org/iderudit/018635ar

DOI : https://doi.org/10.7202/018635ar

Aller au sommaire du numéro

Éditeur(s)

Université Sainte-Anne

ISSN

1498-7651 (imprimé)

1916-7334 (numérique)

Découvrir la revue

Citer cet article

Robichaud, J.-L. (2006). La paroisse et l'église Sacré-Coeur de Saulnierville. Port Acadie, (10-11-12), 129-141. https://doi.org/10.7202/018635ar

\section{Résumé de l'article}

Après un bref aperçu de la construction (1879-1880) et de l'agrandissement (1910) de cette deuxième église construite par l'abbé Jean-Marie Gay, qui est la plus vieille église paroissiale de Clare, et des rénovations qui y ont été apportées suite au renouveau liturgique préconisé par Vatican II, l'auteur examine le rôle de certains curés dans le développement de la vie paroissiale et dans la mise en place d'infrastructures visant le développement spirituel et temporel des paroissiens. Il expose enfin quelques éléments du patrimoine religieux qui, avec l'église, constituent la paroisse Sacré-Coeur de Saulnierville. 
DeuXIÈME PARTIE : L'ÉtAT DES LIEUX DE CULTE

LIEUX DE CULTE DU SUD-OUEST

\section{La paroisse et l'église Sacré-Cœur de Saulnierville}

Jean-Louis Robichaud
Centre provincial de ressources
pédagogiques

Résumé

Après un bref aperçu de la construction (1879-1880) et de l'agrandissement (1910) de cette deuxième église construite par l'abbé Jean-Marie Gay, qui est la plus vieille église paroissiale de Clare, et des rénovations quiy ont été apportées suite au renouveau liturgique préconisé par Vatican II, l'auteur examine le rôle de certains curés dans le développement de la vie paroissiale et dans la mise en place d'infrastructures visant le développement spirituel et temporel des paroissiens. Il expose enfin quelques éléments du patrimoine religieux qui, avec l'église, constituent la paroisse Sacré-Cœur de Saulnierville.

\section{Saulnierville, le village}

L'histoire du village de Saulnierville ressemble beaucoup, pour ses débuts du moins, à celle des premières installations permanentes à la baie Sainte-Marie après le Grand Dérangement de 1755. Suite à l'arrivée de Joseph et Marie Dugas dans la région de l'Anse-des-LeBlanc en 1768, vingt-quatre familles, qui étaient revenues de la Déportation et qui se trouvaient dans la région de Port-Royal, se rendent à la baie SainteMarie en 1769. Parmi celles-ci, notons la présence de René Saulnier et de Marie-Josephe Trahan et de leur famille. Assez rapidement, on commence l'installation sur des terres longeant le littoral de la baie Sainte-Marie en direction du sud. Dès 1768, John Morrison et ses hommes arpentent le district de Clare en allant vers Meteghan. La concession de terres de 1785 accorde les lots 68 à 84 à ceux qui allaient devenir les fondateurs de Saulnierville. Cette concession commence au pont Boudreau et va jusqu'à ce que nous appelons aujourd'hui le chemin à Duffy. Ces terres sont concédées à Joseph, Pierre et Simon Saulnier, Thomas Doucet, Pierre Melanson, Jean-Baptiste Saulnier, Jean Saulnier, Dominique Doucet, William Winnette, Jean-Baptiste Saulnier, fils, Pierre Boudreau, Charles Melanson, Alexandre Thériault, Edmond Robichaud, François à Maza Comeau, Jean Comeau et Joseph Melanson. Ce sont eux et leurs familles que l'on considère comme les fondateurs du village de Saulnierville, 
probablement à cause de René Saulnier, qui semble avoir été le chef de la bande des pionniers, ou en son honneur.

C'est à partir de la deuxième moitié $d u$ xIx ${ }^{\mathrm{e}}$ siècle que Saulnierville commence à connaître un essor économique plus stable, avec la mise en place d'infrastructures plus ouvertes sur le monde extérieur. À partir de 1861, Saulnierville a son propre bureau de poste. Déjà, à cette époque, Saulnierville était le lieu d'arrêt pour la poste à cheval, la stage coach. Il y avait, au coin du chemin qui mène au quai, un hôtel où les gens pouvaient loger pour la nuit. Dans le même coin, il y avait le magasin des Potter. Plus tard, François à Prosper établit un magasin dans le même voisinage. À partir de 1874, le village s'ouvre encore davantage sur le monde extérieur, car c'est alors que débute dans Clare la construction du chemin de fer. C'est tout un monde qui s'ouvre pour les gens de Saulnierville. La poste est alors transportée par voie ferrée. Le chemin de fer permet de communiquer plus directement avec Halifax, pour les rares personnes qui doivent se rendre au Nouveau-Brunswick ou au Québec. Le chemin de fer assure aussi un lien direct et, somme toute, assez rapide avec la petite ville de Yarmouth, où des traversiers permettent d'atteindre les États de la Nouvelle-Angleterre.

Ce qu'il est important de retenir de cette période qui va grosso modo jusqu'à la fin du xix ${ }^{\mathrm{e}}$ siècle, soit plus précisément jusqu'à l'époque de la fondation de la paroisse, c'est l'énorme effort consenti par nos ancêtres pour la réinstallation sur des terres très peu hospitalières, où l'agriculture rapportait peu, mais suffisait quand même à assurer une subsistance. Petits regroupements, petits commerces, petites industries... En fait, nous ne saurions probablement que peu de choses de cette période sans l'apport de la tradition orale, car les gens - et ce, jusqu'à la fondation de la paroisse - ont laissé peu de documents écrits. Il y en a malgré tout quelques-uns. Par exemple, mon arrière-arrière-arrière-arrière-grand-père du côté de ma mère, Jean-Chrysostôme Comeau, décédé en 1805, dans un testament rédigé par l'abbé Sigogne, nous apprend qu'il laisse sa maison, ses terres et ses biens à son épouse Suzanne, d'où le nom donné à la famille... les P'tits Suzanne... Cette maison, qui a plus de deux cents ans, est restée dans notre famille jusqu'à ce qu'elle soit vendue aux Filles de Jésus pour devenir couvent, en 1971.

Mais, en gros, nous avons très peu de documents datant d'avant la fondation de la paroisse. Il y en a, mais il faut savoir les dénicher. Heureusement que des gens comme Lucille Comeau, le père Delbé Comeau, Lina Comeau, Juliette Saulnier, Lucille Amirault, Rose-Marie Thériault et quelques autres ont eu la sagesse de recueillir des documents écrits, mais surtout de recueillir des témoignages de la tradition orale, afin de les consigner à l'écrit avant qu'ils ne se perdent. 


\section{La paroisse et l'église Sacré-Cœur}

Après l'installation définitive des premières familles acadiennes à Saulnierville, celles-ci feront partie de la paroisse Sainte-Marie de la Pointe-de-l'Église à partir de 1799. C'est là qu'elles se rendent, lorsqu'elles le peuvent, pour faire leurs devoirs religieux jusqu'en 1880. Il faut dire cependant que deux choses ont marqué le paysage religieux de Saulnierville avant l'établissement de la paroisse et la construction de l'église Sacré-Cœur.

Premièrement, il est bon de savoir que l'église catholique n'était pas la seule religion pratiquée à la baie à cette époque. En effet, après être venu à la baie Sainte-Marie en 1860 et y avoir trouvé bon accueil, comme les Acadiens le font toujours devant l'étranger, un certain Michel Normanday, pasteur baptiste qui avait des connaissances à Wolfville, achète en 1864 une maison à Saulnierville et y construit une salle qui porte le nom de «Mission acadienne »-en somme, une église baptiste ${ }^{1}$. Isiah Wilson, historien du comté de Digby, nous apprend que le pasteur Normanday réussit à convaincre une dizaine de personnes de la baie de le suivre. Ce qui est moins clair, c'est de savoir s'il s'agissait de catholiques acadiens ou d'anglo-protestants vivant dans la région. Après le départ du pasteur Normanday, il est remplacé par un pasteur Rouleau, mais il faut bien croire que les adeptes de la nouvelle évangélisation se font très rares et, en 1890, la mission est abandonnée. Il faut dire que, pendant les années 1880 et surtout après 1890 , avec l'arrivée des eudistes, les prêtres se font plus nombreux et que leur influence sert grandement à structurer la société sur un modèle catholique, avec tous les mouvements, toutes les associations et, j'ose le dire, avec toutes les contraintes que cela impose.

Quoi qu'il en soit, j'ai l'impression, sans avoir étudié le sujet à fond, que cela doit être plutôt des protestants vivant dans la région qui ont fait partie de cette église baptiste, car les registres paroissiaux sont plutôt silencieux quant à la réintégration de catholiques qui se seraient convertis au protestantisme.

La deuxième observation que je voudrais faire relativement à la vie catholique à Saulnierville avant la fondation de la paroisse est la suivante. Dans les années qui précédent la fondation de la paroisse, la population de Saulnierville grandit rapidement et l'abbé Jean-Marie Gay, alors curé de Sainte-Marie, a pris l'habitude de se rendre à Saulnierville pour célébrer des baptêmes et à l'occasion célébrer la messe. Tout ceci se fait dans la grande maison de mon aïeul maternel, qui a servi d'hôtel, celle de Tom

1. J.-Alphonse Deveau, Clare ou la Ville française, Pointe-de-l’Église, Imprimerie de

l'Université Sainte-Anne, t. I, « Les Premiers cent ans », [sans date], p. 170. 
Saulnier, au pied de la butte du même nom, " grand-père Patate " comme on le nommait. En effet, ma grand-mère Jessie m'a souvent rappelé que l'harmonium qui se trouve dans la sacristie de l'église Sacré-Cœur était celui de sa maison et qu'il servait à exercer les chantres avant la fondation de la paroisse et aussi à faire les frais d'accompagnement lorsque l'abbé Gay se rendait à Saulnierville pour son ministère.

En 1880, donc, est fondée officiellement la paroisse Sacré-Cœur et c'est l'abbé Jean-Marie Gay qui la dessert jusqu'en 1890. Le territoire de la paroisse Sacré-Cœur est borné au nord par le pont Boudreau, qui sépare Saulnierville de Comeauville, et au sud par le pont de la Butte, où commence la paroisse Stella-Maris de Meteghan. Vous aurez sûrement remarqué qu'il s'agit, avec Saint-Alphonse, de la paroisse qui a la plus petite étendue de terre le long du littoral de la baie Sainte-Marie. La paroisse SainteMarie a presque deux fois l'étendue de celle du Sacré-Cœur le long de la baie. La paroisse Sacré-Cœur est cependant très étendue à l'intérieur des terres, avec une quinzaine de chemins qui partent du chemin du roi pour s'enfoncer à l'intérieur des terres. Ces chemins rejoignent de petits cantons tels que la Station-de-Saulnierville, Pipetown, Bangor, la Stationde-Meteghan, Saint-Bénoni et, tout en arrière, un nouveau petit village qui prend forme autour du lac d'Anguilles (ill. 1).

J'ai écrit que la paroisse a été fondée en 1880, mais il faut dire que les travaux de construction de l'église ont débuté au printemps de 1879 et ils ont été menés avec tellement d'entrain que la première messe y a été célébrée à l'automne de la même année par Jean-Marie Gay. Inutile de vous dire que l'église n'était pas complètement terminée, mais elle était, comme les vieux le disent encore, "étanche».

En vue de la construction de l'église, on avait acheté un terrain dans la région du chemin Amirault, mais probablement avec le déplacement de plus en plus marqué de la population vers le sud de la nouvelle paroisse, c'est en 1879 que mesdames Hilaire Boudreau et Ambroise Thériault cèdent à la corporation épiscopale tout le terrain où se trouvent actuellement la salle paroissiale, l'église Sacré-Cœur, le Foyer Gabriel, l'ancien presbytère et aussi, je crois, le terrain sur lequel se trouve l'école Jean-Marie-Gay.

Il existe aujourd'hui quelque confusion par rapport à l'érection de l'église, à son inauguration, à sa bénédiction. D’après les registres et les ouvrages consultés ainsi que les vestiges recueillis, il est possible d'affirmer, comme je l'ai fait plus haut, que l'église a été construite en $1879 .$. Ce sont les notes de Jean-Marie Gay qui l'attestent. De plus, mon frère Richard, qui a travaillé aux rénovations majeures de l'église en 1979, m'affirme être monté jusqu'à la cloche de l'église et, de là, avoir emprunté l'échelle qui monte jusqu'au pied de la croix qui surplombe le clocher. Il me dit qu'au « fi-fin fait » intérieur du pic on avait inscrit la date 


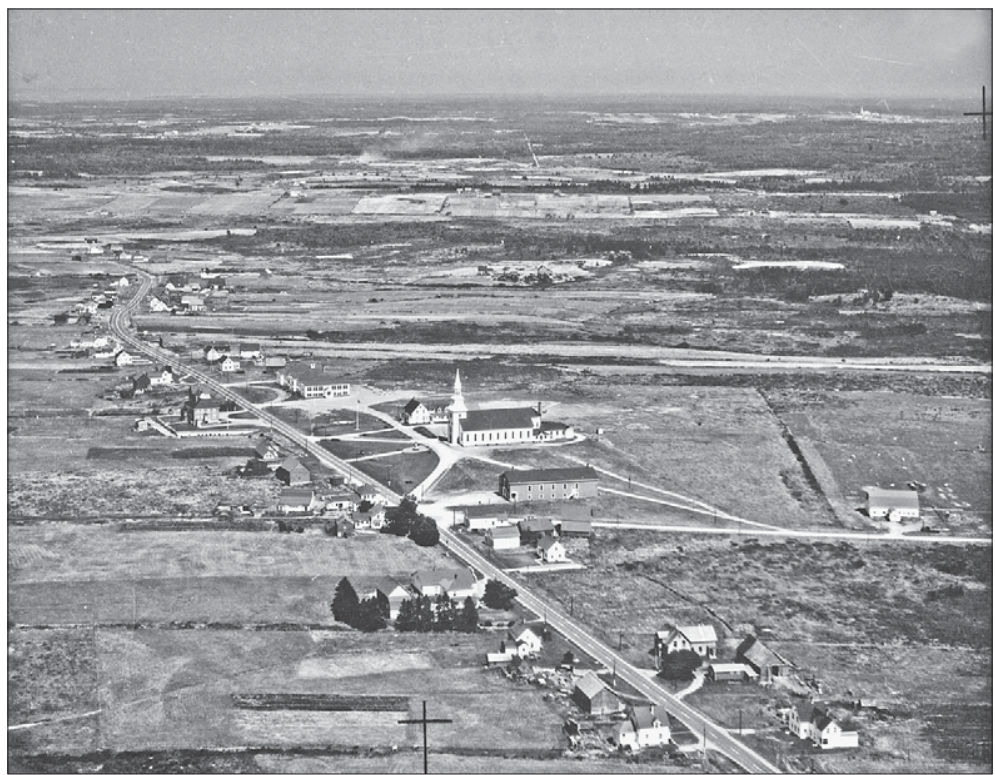

1. Vue aérienne de la paroisse Sacré-Cœur, vers 1960. Collection D. B. Field, Centre acadien, Université Sainte-Anne.

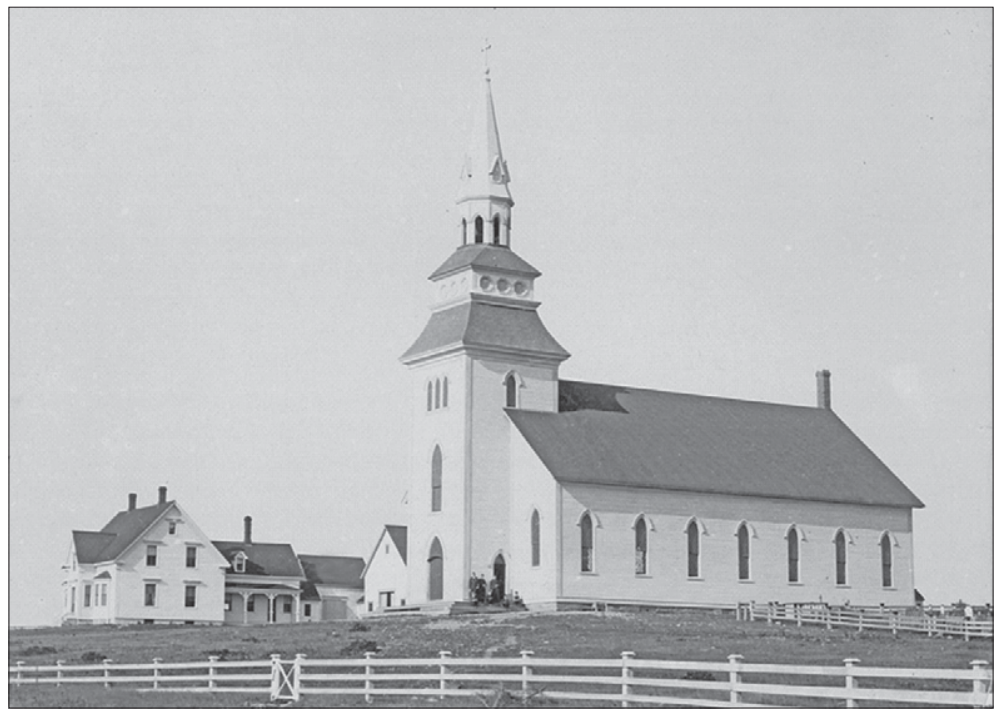

2. Église Sacré-Cœur de Saulnierville, érigée en 1879-1880, Archives de la paroisse, avant 1910. 
de 1879, comme c'était et c'est encore chez nous l'habitude de le faire, par exemple lors des agrandissements de l'église, de ses rénovations, de la construction de la salle paroissiale, des travaux de maçonnerie au cimetière et j'en passe. Quoi qu'il en soit, je pense que le clocher était terminé en 1879, alors si le fait du clocher l'était, je ne vois pas très bien comment le reste de l'église ne l'aurait pas été! La grosse charpente de l'église était terminée, mais bien évidemment pas les travaux de finition intérieure et les décorations. L'abbé Jean-Marie Gay y célèbre la messe de minuit à Noël en 1879.

Tout le bois de construction a été donné par des paroissiens et quelques moulins de la région. La cave a été creusée à la main, comme il se devait à l'époque. Tout le bois a été raboté à la main. Les paroissiens ont donné chacun vingt-cinq jours de travail et ceux qui ne pouvaient s'y rendre se sont fait remplacer. L'abbé Gay est passé de porte en porte pour recueillir des offrandes et il a ramassé une somme considérable d'argent. Il nous dit dans un état de comptes du 26 mai 1883 que l'église a coûté 9386,15 \$, soit 4295,77 \$ en argent pour la construction et le décor, et $5090,00 \$$ en matériel et en travail qui ont été fournis gratuitement ${ }^{2}$. Note intéressante : l'église qui a été bâtie pour quelque 9 000,00 \$ est assurée aujourd'hui pour une valeur de presque $2800000,00 \$$.

La bénédiction et la dédicace de l'église sont faites le 14 septembre 1881 par l'archevêque d'Halifax, Michael Hannan. Participent aussi à la cérémonie trois autres évêques et une quinzaine de prêtres. CamilleAntonio Doucet, dans son livre Une étoile s'est levée en Acadie, nous apprend que c'est le grand patriote acadien $M^{\text {gr }}$ Marcel-François Richard qui prononce le sermon de circonstance ${ }^{3}$. On s'affaire en $1880-1881$ à terminer l'église et à recouvrir tout l'intérieur de plâtre.

Un an jour pour jour après la bénédiction de l'église, soit le 14 septembre 1882, a lieu la bénédiction de la cloche. C'est le père Camille Lefebvre, supérieur du Collège Saint-Joseph de Memramcook, qui prononce le sermon de circonstance en présence de $M^{\text {gr }}$ Power, administrateur de l'archidiocèse, de plusieurs prêtres et d'une foule de fidèles. Cette cloche pèse 650 livres et ce sont des paroissiens et paroissiennes qui ont donné des pièces d'argent qu'on a fait fondre afin de les incorporer à la cloche, ce qui lui donne son ton si distinctif. La fonte de la cloche a été payée par cinq dames de la paroisse, qui sont connues comme les marraines de la cloche. Malheureusement, aucun renseignement trouvé à ce jour ne nous permet de les identifier.

2. Lucille Comeau, 'Sont les gens de Saulnierville, Yarmouth, Imprimerie Lescarbot, 1980, p. 27.

3. Camille-Antonio Doucet, Une étoile s'est levée en Acadie, Charlesbourg, Éditions du Renouveau, 1973, p. 71. 
Entre 1880 et 1890, l'abbé Jean-Marie Gay se rend tous les deux dimanches à Saulnierville célébrer la grand-messe, célébrant à la Pointede-l'Église les autres dimanches. Dès le début de la paroisse, on signale la qualité du chant aux offices. Déjà un article de L'Évangéline en fait état en 1887 et on mentionne le talent des organistes, de la direction, des solistes. Ce genre d'article reviendra souvent dans L'Évangéline et plus tard dans le Petit Courrier.

\section{L'édifice de l'église}

Alors, quelle était cette église érigée en 1879-1880... De quoi avaitelle l'air? Une photographie de l'église des débuts, trouvée dans les archives de la paroisse, nous éclaire (ill. 2). On remarque que le clocher était sensiblement différent, avec des décorations plus raffinées que celle que l'on peut observer aujourd'hui. Également à noter : l'absence de vitraux. Ce sont de grandes fenêtres avec de la vitre taillée en losanges, un peu comme dans l'ancienne église Saint-Bernard. Des verrières les remplaceront en 1896. De plus, l'église est beaucoup plus petite en longueur que celle d'aujourd'hui. En guise de sacristie, il n'y a qu'une petite rallonge de vingt-six pieds sur onze pieds. La photographie nous permet aussi d'apercevoir le premier cimetière de la paroisse, qui était situé en arrière et du côté sud de l'église. Ce cimetière sera abandonné à l'occasion de l'agrandissement de l'église en 1910. Plusieurs se demandent pourquoi on a abandonné ce cimetière en faveur de la « butte à grave ", site actuel du cimetière - le " parc à Eustache », comme on le désignait autrefois ${ }^{4}$. C'est sans doute l'origine de l'expression utilisée par les vieillards de la paroisse lorsqu'ils disent que nous finirons tous par "manger des pissenlits par les racines dans le parc à Eustache »! Mais je reviens à ma question : pourquoi changer le site du cimetière?

Voici la réponse. La butte, où est située l'église Sacré-Cœur et qui la met si bien en évidence à des kilomètres à la ronde, renferme une nappe d'eau souterraine qui est puissante et presque à la surface du sol. Au sous-sol de l'église, on a aménagé, dès le début, un canal où coule un petit ruisseau à longueur d'année et qui, mesurant environ un mètre de profondeur, traverse l'église du nord-est au sud-ouest. Depuis une vingtaine d'années, ce canal a été comblé de pierres grossières et recouvert d'un gravier plus fin, le tout permettant au petit ruisseau de continuer à couler. C'est cette présence de l'eau presque à la surface de la terre qui a mené à l'abandon du premier cimetière. D'après mon grand-père, lorsqu'une personne décédait, on creusait une fosse et au

4. Delbé Comeau, Mémoires 1905-1949, Yarmouth, Éditions Lescarbot, [sans date], p. 63. 
moment de l'enterrement, il y avait toujours soixante ou soixante-quinze centimètres d'eau dans la fosse et il fallait mettre de grosses pierres sur le cercueil pour le faire "caler », comme on disait, et beaucoup de gens s'objectaient au fait qu'il fallait " noyer » les morts au moment de les mettre en terre.

Ainsi, en 1910, on récupère les ossements identifiables et on les transfère au nouveau cimetière. Certains racontent, des années après, que le père Cantin, le curé de l'époque, aurait été aperçu un soir en train de renverser les pierres tombales des défunts de familles qui s'objectaient au déménagement, mais cela reste à voir, car le père Cantin semble avoir été bien estimé par la majorité des gens de Saulnierville même s’il était souvent très rigide.

Dix ans après la fondation de la paroisse, l'abbé Jean-Marie Gay réussit à convaincre l'archevêque d'Halifax, $M^{\text {gr }} 0$ 'Brien, de faire venir les pères eudistes pour fonder ce qui deviendra le Collège Sainte-Anne. Afin d'assurer les revenus nécessaires aux pères de la congrégation, l'abbé Gay cède ses deux paroisses, Sainte-Marie et Sacré-Cœur, aux eudistes. Ainsi, à partir de 1890 jusqu'à tout dernièrement, ces deux paroisses seront dirigées par les pères de la Congrégation de Jésus et Marie. Dans les deux cas, au début de la fondation, les pères ne résidaient pas au presbytère, mais en communauté, au collège. Ils se rendaient dans les deux églises pour célébrer les offices et les sacrements. Cette situation crée beaucoup de tensions, surtout à Saulnierville, où les gens souhaitaient avoir un curé résidant. Pendant la cure du père Blondel de 1903 à 1905, ce dernier tente en vain de résider à Saulnierville, car le père Dagnaud, supérieur du collège, s'y oppose fermement. Après le départ de ce dernier en 1908, les choses changent et, lorsque Louis-Marie Cantin devient curé du SacréCœur, en 1908, il élit domicile au presbytère de Saulnierville construit pendant la cure de Jean-Marie Gay en 1884.

Il faut ici signaler un événement important dans la vie de la paroisse Sacré-Cœur au tournant du xxe siècle : la première messe de Désiré Comeau, premier prêtre acadien avec Édouard LeBlanc de Saint-Bernard, tous deux natifs de la baie Sainte-Marie. Désiré Comeau est ordonné prêtre le 3 juillet 1898, quatre jours après Édouard LeBlanc. La première messe de Désiré Comeau à Saulnierville donne lieu à de grandes réjouissances dans la jeune paroisse. Depuis cette date, six autres hommes de la paroisse sont devenus prêtres; trois seront séculiers et trois eudistes. Une quarantaine de femmes sont devenues religieuses au sein de sept congrégations religieuses différentes. Parmi ceux qui deviendront eudistes, Jules et Léger Comeau seront appelés à assumer des charges importantes au sein de la communauté, à titre de recteurs de collèges et de grands séminaires. 
En 1908 arrive donc à la cure de Saulnierville le père Louis-Marie Cantin, eudiste français, qui y restera jusqu'en 1921. C'est lui qui entreprend en 1910 l'agrandissement de l'église. Il faut dire que, à ce moment, l'église est sensiblement remaniée. On y ajoute cent soixante-dix places en l'allongeant vers le sud-ouest, on aménage un vrai sanctuaire, on installe douze nouveaux vitraux et on ajoute une sacristie mesurant cinquante pieds sur trente-quatre pieds servant de chapelle aux offices pendant la semaine. L'église telle qu'on la connaît aujourd'hui mesure cinquante pieds de large sur cent quatre-vingt-dix-sept pieds de long, si on compte la sacristie. La croix du clocher s'élève à environ cent pieds au-dessus du niveau du sol. Tous les travaux de 1910, comme ceux de 1879-1880 d'ailleurs, sont faits principalement par les gens de la paroisse, qui font don du matériel et du labeur, et le tout est réalisé sans dettes (ill. 3, 4).

Deux faits à signaler en ce qui concerne l'architecture de l'église SacréCœur. Nous avons encore à la paroisse les plans de l'église, réalisés par un architecte, qui ont été quelque peu modifiés. Il faut dire que, lorsque Jean-Marie Gay décide de construire une église à Saulnierville, il reprend le modèle de l'église qu'il a fait construire pendant sa cure à Wedgeport, en 1867. Le plan extérieur est exactement le même : fenêtres et portes, sauf le clocher, mêmes arches gothiques et colonnes à l'intérieur et j'en passe. L'église de Wedgeport sera agrandie et radicalement transformée par le père Jean Deveau de la Rivière-aux-Saumons en 1923.

Autre fait à noter : lorsque le père Cantin fait agrandir l'église SacréCœur en 1910, il fait venir un ébéniste du Québec pour faire les autels. Malheureusement, nous ne connaissons pas son nom, mais nous savons que c'est lui qui aménage les trois autels d'alors, dont le maître-autel dédié au Sacré-Cœur avec les deux disciples du Sacré-Cœur, saint Jean-Eudes et sainte Marguerite-Marie Alacoque. Il construit aussi deux autels latéraux maintenant disparus, celui de droite dédié à la sainte Vierge offert par les Enfants de Marie et celui de gauche dédié à sainte Anne offert par les dames de la congrégation du même nom. Il faut croire que ces autels sont très prisés à l'époque, car on embauche le même artiste-ébéniste pour reproduire en plus petit les trois mêmes autels pour l'église SainteAgnès de Quinan. En passant, il est intéressant de noter que la paroisse Sacré-Cœur conserve plusieurs cahiers de notes et de procès-verbaux des Enfants de Marie et des Dames de Sainte-Anne qui nous renseignent sur les activités de ces associations et, par extension, sur la vie de la paroisse de la fin du xIx ${ }^{e}$ siècle jusqu'au début des années 1960.

De 1921 à 1933, la paroisse est administrée par deux eudistes français, LeQuéméner et Schreiber. C'est ce même père Schreiber qui, avec l'aide de Denis Gaudet de Saulnierville, exécute les belles boiseries de la sacristie, ainsi que le magnifique chandelier pascal qui est encore en 


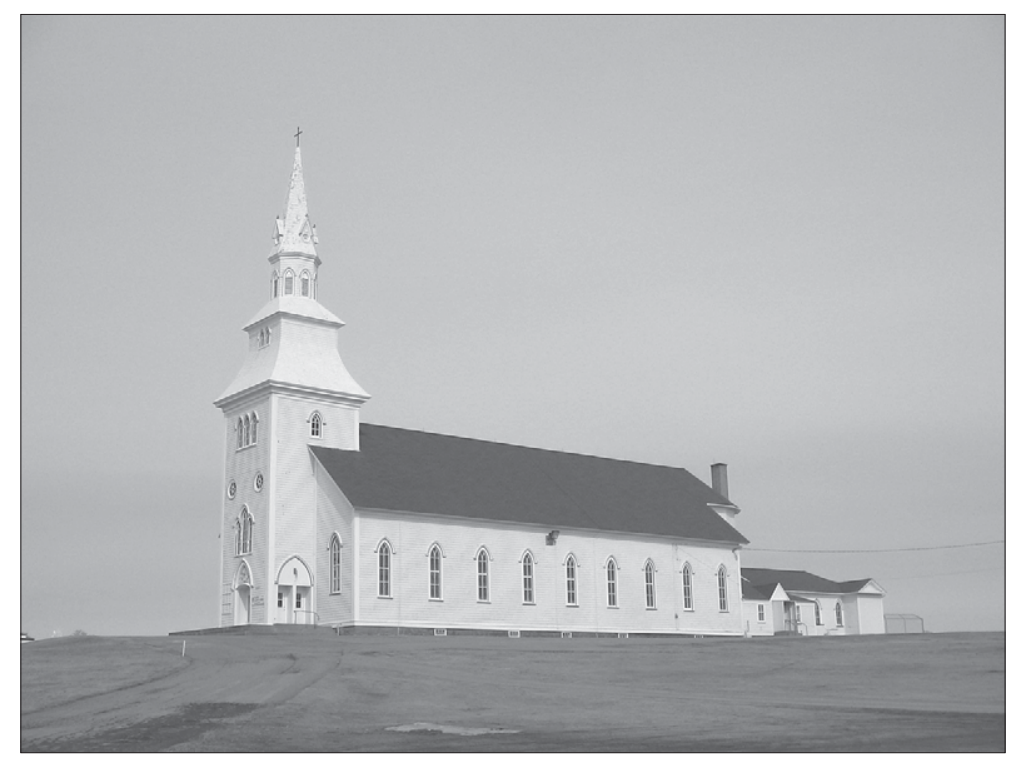

3. Église Sacré-Cœur de Saulnierville actuelle. Photographie de Jean-Pierre Pichette, 2006.

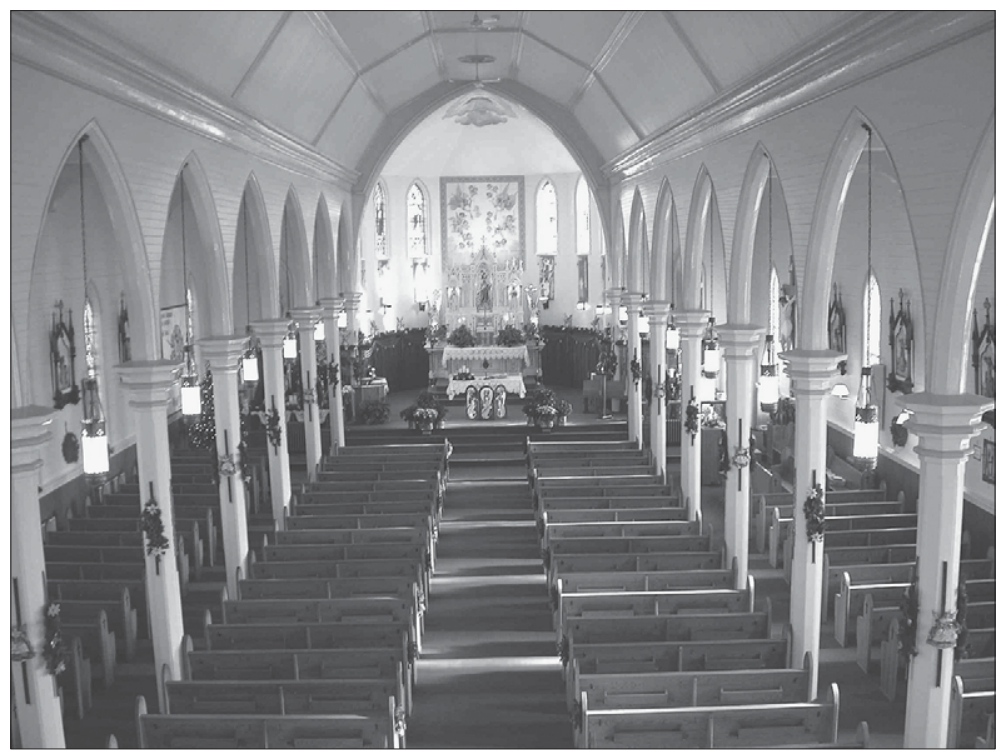

4. Intérieur de l'église Sacré-Cour de Saulnierville. Photographie de Jean-Louis Robichaud, 2006. 
usage aujourd'hui (ill. 5). C'est en 1933 que la paroisse reçoit son premier curé acadien, le père eudiste Jovite à Anthime Doucet, de la Pointe-àMajor. Les archives des eudistes renferment des cahiers, des notes et la correspondance du père Jovite, mais il s'agit plutôt du petit train-train de la vie paroissiale pendant la Grande Dépression et les quatre premières années de la Deuxième Guerre mondiale alors que les activités d'envergure étaient plutôt réduites.

Arrive alors en 1943 à la cure du Sacré-Cœur le père eudiste Thomas Castonguay, ancien élève du Collège Sainte-Anne et ancien curé de la Pointe-de-l'Église et des Concessions. Commence alors et cela, jusqu'à son départ en 1964, la plus grande période d'expansion de la paroisse, sur tous les plans : travaux majeurs à l'église, à l'extérieur et surtout à l'intérieur, rénovations majeures au presbytère, construction de la salle paroissiale, construction de l'école Gay, venue des Filles de Jésus et construction du couvent, fondation de la Caisse populaire et bien d'autres choses encore. C'est le père Castonguay qui fait venir de Montréal l'artiste Chabauty, à qui nous devons la murale au fond du sanctuaire, tout en arrière du maître-autel.

Le concile Vatican II apporte des changements importants au niveau du culte et de la pratique religieuse, nul n'a besoin de le rappeler. Ce qui est moins visible, mais qui est tout aussi important, c'est la manière dont les changements liturgiques sont intégrés à la vie religieuse de la paroisse Sacré-Cœur. En 1965, le curé de l'époque décide d'implanter sans tarder, mais également sans consultation ni préparation des fidèles, certaines réformes liturgiques avancées par les pères du concile.

Ainsi, d'un dimanche à l'autre, les paroissiens et paroissiennes du Sacré-Cœur arrivent à l'église pour trouver le sanctuaire radicalement transformé. Pendant la semaine, sur ordre du curé, on a enlevé et brûlé les deux autels latéraux, on a installé un autel face au peuple, on a supprimé la chaire, on a installé un tout petit ambon et toute la liturgie de la messe est proclamée en français... tout cela sans que personne n'en soit averti. Mais ce qui est pire encore, c'est que le curé en question décide de son propre chef de vendre à des antiquaires du Québec tous les éléments du patrimoine qu'il juge inutilisables dans la nouvelle liturgie. Ainsi s'envolent lampes du sanctuaire, calices, ciboires, ostensoirs, candélabres, chandeliers, encensoirs et j'en passe. Il est inimaginable aujourd'hui, avec l'existence de conseils paroissiaux, que telle chose puisse se reproduire et avec raison. Mais on est bien obligé, hélas, de se résigner à constater simplement la disparition de ces éléments du patrimoine qui n'avaient été acquis, nous pouvons l'imaginer, que par le dur travail de nos ancêtres. 


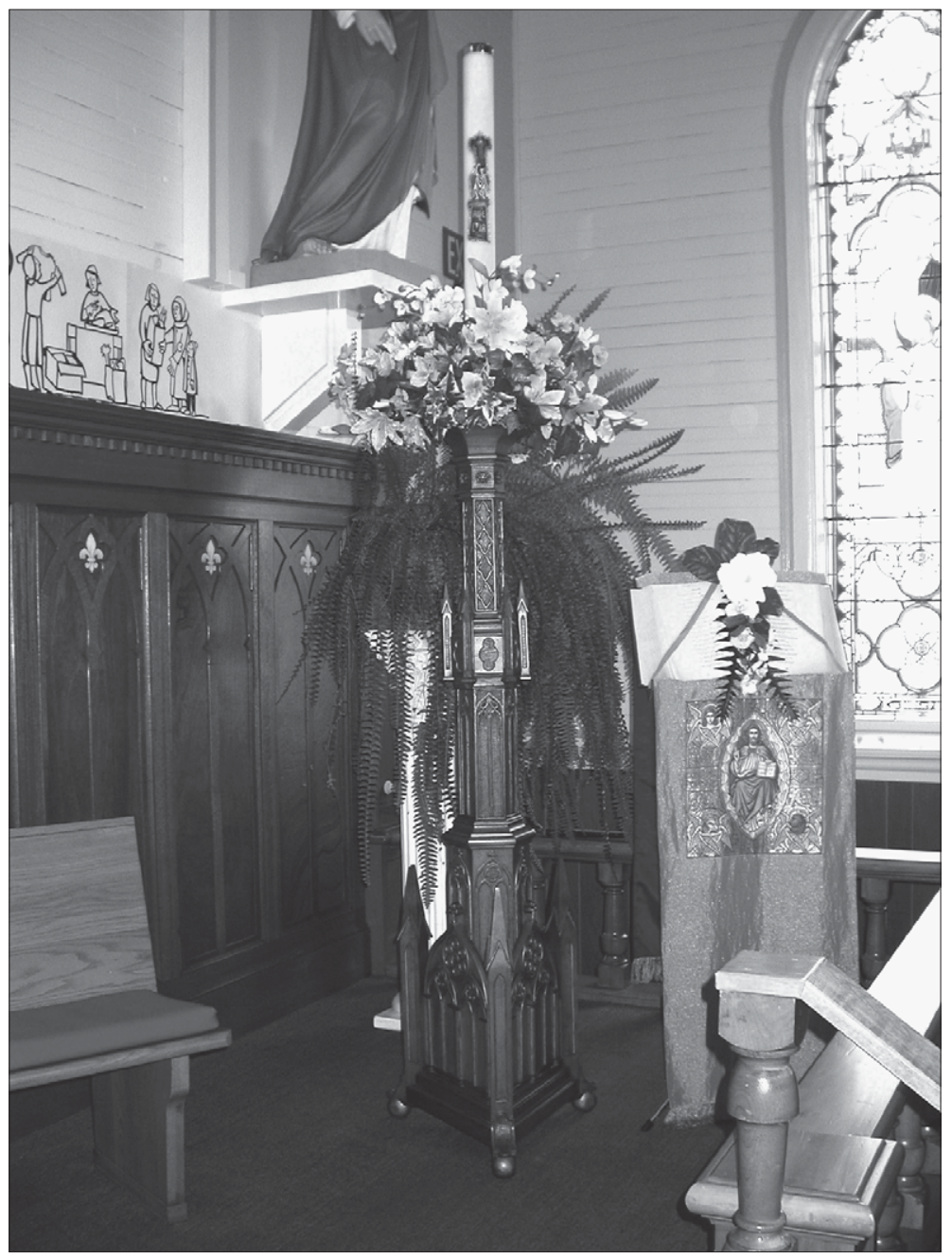

5. Église Sacré-Cour de Saulnierville, chandelier pascal exécuté par l'eudiste Schreiber et Denis Gaudet avant 1933. Photographie de Jean-Louis Robichaud, 2006. 
Cependant, tout n'est pas noir dans le tableau des éléments du patrimoine. La paroisse Sacré-Cœur de Saulnierville avait en sa possession un grand lectionnaire datant des années 1635, je pense. Heureusement que les antiquaires ne s'intéressaient qu'à l'argenterie et aux dorures, car le lectionnaire a survécu! Il a depuis été prêté et déposé au musée religieux de la paroisse Sainte-Marie, où il peut être mis en valeur, plutôt que de rester caché dans une armoire à Saulnierville. Il me semble qu'il y a là un heureux précédent, dont les paroisses de la baie Sainte-Marie pourraient s'inspirer. Pourquoi ne pas déposer au musée les objets du culte qui ne sont plus en usage et qui font partie du patrimoine de la paroisse? Ainsi, ces objets, tout en demeurant propriété de la paroisse, pourraient être mis en valeur dans le cadre des activités et des programmes du musée, car, me semble-t-il, si un patrimoine caché constitue tout de même un patrimoine, sa mise en valeur est ce qui le rend réellement vivant.

Mais revenons à la paroisse et à l'église Sacré-Cœur. À partir de 1975, le presbytère cesse d'être la résidence du curé, car toute la communauté eudiste de la région a élu domicile à l'ancienne auberge Riverside Inn à la Butte, une année avant le transfert du collège à une administration laïque en 1971. La paroisse Sacré-Cœur connaît un grand regain de vie pendant la cure de l'eudiste Claude Côté, de 1975 à 1989. Des améliorations et des réparations majeures de l'ordre de plus de 200000,00 \$ sont apportées à l'église, à la salle et au cimetière, et l'ancien presbytère est démoli. Les mouvements paroissiaux connaissent un regain de vie extraordinaire et les jeunes prennent une part très active à la vie de la paroisse.

Aujourd'hui, la paroisse Sacré-Cœur compte environ 2300 personnes. Ses édifices sont en bon état, grâce aux investissements des quarante dernières années, et ils sont évalués à 3500000,00 \$. Sept comités et une religieuse ainsi qu'une secrétaire assistent le nouveau curé, l'abbé Edgar Mvubu, dans l'administration de la paroisse. À mon avis, le patrimoine vivant le plus important de la paroisse Sacré-Cœur est sans doute le peuple de Dieu, en marche vers l'accomplissement de la mission.

Il est évident, néanmoins, que la paroisse religieuse n’occupe plus la place qu'elle avait autrefois. Même si la pratique religieuse ne se situe plus que dans les dix-huit à vingt pour cent, toute la population réclame les services religieux en temps voulu : baptêmes, premiers pardons et premières communions, mariages, anniversaires, funérailles, éducation de la foi, action sociale, entretien et agrandissement du cimetière... ce qui pose des défis grandissants. Heureusement qu'il y a encore des gens à qui la vie de la communauté tient à cœur et qui continuent de construire cette communauté dans un esprit de partage et d'entraide. 